\title{
Hand-to-face contact behaviors during indoor activities in daily life among Korean adults: an observational pilot study using videotaping
}

\author{
Hyang Soon Oh'1, Mikyung Ryu ${ }^{2}$, Youngran Yang ${ }^{3}$ \\ ${ }^{1}$ Department of Nursing, College of Life Science and Natural Resources, Sunchon National University, Suncheon, Korea; ${ }^{2}$ Department of Nursing, \\ College of Nursing and Public Health, Daegu University, Daegu, Korea; ${ }^{3}$ Research Institute of Nursing Science, Jeonbuk National University \\ College of Nursing, Jeonju, Korea
}

OBJECTIVES: Hand-to-face contact (HFC) is the major route for the self-inoculation of pathogens. This study aimed to describe the characteristics of HFC behaviors among Korean adults during indoor activities.

METHODS: Thirty participants were enrolled in the study, and 2 hours of videotaped data were collected from each participant. Contact data were recorded by examining the frequency and duration of HFC on the videos. Three training sessions were conducted for 2 readers to ensure the accuracy and reliability of videotape reading. Re-reading and verification of selected video data were performed to confirm intrapersonal and interpersonal validity. Contact exposure (CE) was determined by multiplying the contact frequency $(\mathrm{CF})$ by the contact duration $(\mathrm{CD})$ to quantify the intensity of contact during the observation time (2 hours).

RESULTS: A total of 3,007 HFCs (1,305 mucous membrane contacts and 1,702 non-mucous membrane contacts) were observed for 60 person-hours. The median CF (person/2 hr) of the mucous membranes (eye; 4.0, nose; 15.5, mouth; 16.5) was 39.5/person and the median total CD was $177.0 \mathrm{sec} /$ person. The median CE (frequency-duration/sec/person) was 5,795.0 (mouth: 1,356.0, nose: 600.0, eye: 57.5 ).

CONCLUSIONS: This study showed that the mouth and nose were the most frequent exposure sites for HFC. Avoiding habitual HFC, awareness of self-inoculation by HFC, and vigorous hand hygiene should be strengthened to prevent the spread of infections.

KEY WORDS: Activities of daily living, Cross infection, Disease transmission, Hand hygiene, Contact tracing, Touch

\section{Correspondence: Mikyung Ryu}

Department of Nursing, College of Nursing and Public Health, Daegu University, 33 Seondang-ro 50-gil, Nam-gu, Daegu 42400, Korea E-mail:ryumk@daegu.ac.kr

Received: Dec 16, 2020 / Accepted: Apr 22, 2021 / Published: Apr 22, 2021

This article is available from: https://e-epih.org/

(C) This is an open-access article distributed under the terms of the Creative Commons Attribution License (https://creativecommons.org/licenses/by/4.0/), which permits unrestricted use, distribution, and reproduction in any medium, provided the original work is properly cited.

(C) 2021, Korean Society of Epidemiology

\section{INTRODUCTION}

Many kinds of infectious diseases, including recent 21st-century diseases such as severe acute respiratory syndrome (SARS) and Middle Eastern respiratory syndrome (MERS), have threatened public health despite meaningful developments in infection prevention techniques. The most frequent mode of transmission of such infectious diseases is contact with contaminated hands; therefore, strict hand hygiene and avoidance of hand-to-face contact (HFC) are strongly recommended to prevent the transmission of infections [1,2].

HFC is the major route for self-inoculation of pathogens [3]. When someone touches his or her eyes, nose, or mouth with contaminated hands, self-inoculation of pathogens can occur. Self- 
inoculation is a type of hand contact transmission where a person's contaminated hands make subsequent contact with other sites on the person's body, introducing contaminated material [4]. Self-inoculation via HFC in individuals leads to further opportunities for transmission; thus, it can constitute a potential transmission route of infectious diseases in the community $[4,5]$. This behavior can be particularly relevant during pandemics, such as SARS, MERS, and novel influenza A (H1N1) [6].

Studies on self-inoculation via HFC have been conducted for respiratory infections $[5,7]$ and Staphylococcus aureus infections [8] among healthcare workers [9] and biosafety workers [10]. A substantial proportion of human respiratory tract infections are thought to be transmitted via contact between contaminated hands and the mucous membranes of the mouth, eyes, and/or nose, and a key risk factor for infection transmission is the rate of hand contact with these areas, termed "target facial mucous membranes" [5]. Respiratory viral tract infections such as rhinovirus $[7,11]$ and H1N1 [6] have been reported to be transmitted by self-inoculation. One study reported an HFC rate per hour of 15.7 and proposed an equation to predict the transmission of respiratory diseases [5].

Therefore, understanding the characteristics of HFC is important for estimating infectious pathogen exposure in addition to chemical exposure [12]. However, few studies have described the frequency or patterns of HFC in terms of infectious exposures, despite strong recommendations to avoid HFC [13]. Studies on HFC have mostly focused on chemical exposures [12,14]. Additionally, the reported characteristics of HFCs in previous studies have varied $[4,9,10,12,14,15]$.

Moreover, studies on the patterns and frequencies of HFC in the Korea have not been conducted yet, as a means of self-inoculation of infectious pathogens in Korea. Therefore, evidence on ways to avoid HFC as a means of infection prevention is scarce. This study conducted a brief survey to describe the frequency, duration, and intensity of HFC among Korean adults during daily indoor activities as a pilot study to provide evidence for estimating pathogen exposure according to HFC behavior.

\section{MATERIALS AND METHODS}

\section{Study setting and participants}

From January 14, 2018 to February 12, 2018, participants were selected by convenience sampling. A total of 30 Korean adults aged $\geq 20$ years participated, including 10 university students from one university, 10 university students, faculty members, and faculty assistants from another university, and 10 participants from a church congregation. We first explained the purpose and method of this study to the directors of the institutions. We then explained the requirements for videotaping indoor activity data during daily life to the participants. Participants who voluntarily agreed to take part in this study were enrolled and their consent was obtained for videotaping their activities. The participants understood and agreed that the researchers would measure their behaviors from the recorded tapes, which would be kept confiden- tial to protect their privacy. To reduce the possibility of consciously limiting HFC behavior by the participants during the videotaping, we did not precisely inform them about the specific HFC behaviors being observed for the purposes of the study. After recording their behaviors by videotaping, participants were then advised of the precise aspects of HFC shown on their behavior recordings. They subsequently consented to our measurement of HFC after the video recordings. The videotaping locations were chosen to facilitate indoor activities in a daily living environment, such as a lecture room for university students, workplace offices for faculty members and faculty assistants, and a church chapel for church congregation members. Participants' behaviors were recorded via videotape for 2 hours each (60 person-hours) and were measured during the most active time on a day that was convenient to participants. Participants performed their daily routine tasks involving indoor activities (such as lectures, discussions, reading, writing, computer work, worshipping, or Bible study) as usual during the videotaping.

\section{Data collection/procedure}

Data on the contact frequency (CF) of HFCs, contact duration (CD) of HFCs, and HFC areas were recorded for each participant in a standardized Excel form. The reading time to check each CD was recorded in the form of start minute, end minute, start second, and end second (referring to the timestamp at the bottom of the video recording) to avoid missing time. If the reading time was missed, the video playback was checked to prevent data loss. Contact areas were observed, and HFC was classified in terms of whether it involved contact with mucous membranes.

\section{Data accuracy and reliability}

To ensure the accuracy and reliability of the video reading, we secured 2 video readers to enable cross-verification, and we conducted 3 training sessions. To reduce intrapersonal reading errors, $4 \%$ of the data entered by each reader was re-read and checked for contact areas, CF, and CD to ensure a match of more than $90 \%$. The minimization of interpersonal reading errors was confirmed by an agreement of more than $90 \%$ in approximately $10 \%$ of randomly selected readings taken by the other reader.

\section{Definition of terms}

Mucous membrane contacts were defined as HFCs involving the eye, nose, and mouth, and non-mucous membrane contacts were defined as HFCs involving the skin of the head, forehead, chin, cheek, and ear.

We defined the contact exposure (CE) index by multiplying $\mathrm{CF}$ and $\mathrm{CD}$ to estimate HFC exposure. CE was defined to quantify the total number of HFCs during 2 hours of observation.

\section{Statistical analysis}

Continuous variables and categorical variables were presented as mean \pm standard deviation or median (25th percentile; 75 th percentile), interquartile range, and frequencies and percentages, 
respectively. We used the Kruskal-Wallis test and the Fisher exact test to compare the demographic characteristics of the 3 groups recorded in different settings (chapel, classroom, and office) according to the data type. The Dunn post-test and Kruskal-Wallis test were used for multiple comparisons to investigate the differences in contact indices between chapel, classroom, and office settings. Statistical significance was set at $\mathrm{p}<0.05$. Data analyses were performed using R 3.3.3 (version 3.15) for Windows ( $R$ Foundation for Statistical Computing, Vienna, Austria).

\section{Ethics statement}

All procedures performed in this study involving human participants followed the ethical standards of the appropriate institutional research committee (IRB No. 1040173-201712-HR-033-02) and were conducted according to the 1964 Helsinki Declaration and its later amendments or comparable ethical standards. Informed written consent was obtained from all individual participants in this study before video recording and after video recording.

\section{RESULTS}

\section{General characteristics of participants}

The average age of the 30 participants was 41.0 years. Those in their 20 s accounted for $43.3 \%$; $63.3 \%$ were female, $51.7 \%$ were high school graduates, and $27.6 \%$ were university students (Table 1 ). Table 1 shows the general characteristics of the participants in each indoor setting (chapel, classroom, and office).

Table 1. General characteristics of participants $(n=30)$

\begin{tabular}{|c|c|c|c|c|c|}
\hline Characteristics & Total & Chapel $(n=10)$ & Classroom $(n=10)$ & Office $(n=10)$ & $p$-value \\
\hline Age (yr) & & & & & $<0.001$ \\
\hline $20-29$ & $13(43.3)$ & $0(0.0)$ & $10(100.0)$ & $3(30.0)$ & \\
\hline $30-59$ & $10(33.3)$ & $4(40.0)$ & $0(0.0)$ & $6(60.0)$ & \\
\hline$\geq 60$ & $7(23.3)$ & $6(60.0)$ & $0(0.0)$ & $1(10.0)$ & \\
\hline Mean \pm SD (median) & $41.0 \pm 18.5(43.0)$ & $60.0 \pm 9.6(60.0)$ & $21.4 \pm 1.5(21.0)$ & $41.6 \pm 13.3(43.5)$ & $<0.001$ \\
\hline Sex & & & & & 0.510 \\
\hline Male & $11(36.7)$ & $5(50.0)$ & $4(40.0)$ & $2(20.0)$ & \\
\hline Female & $19(63.3)$ & $5(50.0)$ & $6(60.0)$ & $8(80.0)$ & \\
\hline Marital status & & & & & $<0.001$ \\
\hline Single & $14(46.7)$ & $0(0.0)$ & $10(100)$ & $4(40.0)$ & \\
\hline Married & $16(53.3)$ & $10(100)$ & $0(0.0)$ & $6(60.0)$ & \\
\hline Education & & & & & $<0.001$ \\
\hline High school and under & $15(51.7)$ & $7(77.8)$ & $8(80.0)$ & $0(0.0)$ & \\
\hline College (undergraduate) & $8(27.6)$ & $2(22.2)$ & $2(20.0)$ & $4(40.0)$ & \\
\hline Graduate school & $6(20.7)$ & $0(0.0)$ & $0(0.0)$ & $6(60.0)$ & \\
\hline Occupation & & & & & $<0.001$ \\
\hline None & $5(16.7)$ & $5(50.0)$ & $0(0.0)$ & $0(0.0)$ & \\
\hline Student & $11(36.7)$ & $0(0.0)$ & $10(100)$ & $1(10.0)$ & \\
\hline Officer & $5(16.7)$ & $2(20.0)$ & $0(0.0)$ & $3(30.0)$ & \\
\hline Sales/service & $1(3.3)$ & $1(10.0)$ & $0(0.0)$ & $0(0.0)$ & \\
\hline Health care provider & $1(3.3)$ & $0(0.0)$ & $0(0.0)$ & $1(10.0)$ & \\
\hline Others & $7(23.3)$ & $2(20.0)$ & $0(0.0)$ & $5(50.0)$ & \\
\hline Economic activity & & & & & $<0.001$ \\
\hline Unemployed (including students) & $16(53.3)$ & $5(50.0)$ & $10(100)$ & $1(10.0)$ & \\
\hline Employed & $14(46.7)$ & $5(50.0)$ & $0(0.0)$ & $9(90.0)$ & \\
\hline Household income/mo ( $\left.10^{4} \mathrm{KRW}\right)$ & & & & & 0.008 \\
\hline$<300$ & $7(25.0)$ & $5(62.5)$ & $2(20.0)$ & $0(0.0)$ & \\
\hline $300-<500$ & $8(28.6)$ & $3(37.5)$ & $3(30.0)$ & $2(20.0)$ & \\
\hline $500-<1,000$ & $11(39.3)$ & $0(0.0)$ & $5(50.0)$ & $6(60.0)$ & \\
\hline$\geq 1,000$ & $2(7.1)$ & $0(0.0)$ & $0(0.0)$ & $2(20.0)$ & \\
\hline Residence & & & & & 0.012 \\
\hline Metropolitan & $18(60.0)$ & $8(80.0)$ & $8(80.0)$ & $2(20.0)$ & \\
\hline City or small town & $12(40.0)$ & $2(20.0)$ & $2(20.0)$ & $8(80.0)$ & \\
\hline
\end{tabular}

Values are presented as number (\%).

$\mathrm{SD}$, standard deviation; KRW, Korean won.

1The Kruskal-Wallis test was used for continuous variables and the Fisher exact test for categorical variables. 
Table 2. Descriptive statistics of contact indices ( $n=30,2$-hour observation time)

\begin{tabular}{|c|c|c|c|c|c|c|}
\hline Variables & $\begin{array}{l}\text { Observed contacts } \\
(\mathrm{n}=3,007)\end{array}$ & Mean $\pm S D$ & 1Q & Median & $3 Q$ & IQR \\
\hline \multicolumn{7}{|l|}{ Contact frequency (n/person) ${ }^{1}$} \\
\hline Mucous membranes & 1,305 & $43.5 \pm 19.4$ & 33.5 & 39.5 & 51.5 & 18.0 \\
\hline Eye & 180 & $6.0 \pm 5.6$ & 2.0 & 4.0 & 9.5 & 7.5 \\
\hline Nose & 541 & $18.0 \pm 13.8$ & 9.3 & 15.5 & 19.5 & 10.3 \\
\hline Mouth & 584 & $19.5 \pm 16.2$ & 8.0 & 16.5 & 27.0 & 19.0 \\
\hline Non-mucous membranes & 1,702 & $56.7 \pm 40.4$ & 26.0 & 49.0 & 74.5 & 48.5 \\
\hline Head & 835 & $27.8 \pm 29.0$ & 7.3 & 13.0 & 48.5 & 41.3 \\
\hline Forehead & 80 & $2.7 \pm 3.4$ & 0.0 & 2.0 & 3.8 & 3.8 \\
\hline Chin & 326 & $10.9 \pm 12.6$ & 3.3 & 6.0 & 14.8 & 11.5 \\
\hline Cheek & 372 & $12.4 \pm 17.0$ & 3.3 & 6.5 & 14.0 & 10.8 \\
\hline Ear & 89 & $3.0 \pm 3.2$ & 1.0 & 2.0 & 3.8 & 2.8 \\
\hline \multicolumn{7}{|l|}{ Contact duration (sec/person) ${ }^{2}$} \\
\hline Mucous membranes & 1,305 & $232.9 \pm 227.4$ & 111.5 & 177.0 & 256.5 & 145.0 \\
\hline Eye & 180 & $30.4 \pm 35.5$ & 7.0 & 17.0 & 39.5 & 32.5 \\
\hline Nose & 541 & $73.3 \pm 61.6$ & 30.0 & 42.5 & 99.8 & 69.8 \\
\hline Mouth & 584 & $129.3 \pm 215.0$ & 19.0 & 72.5 & 124.8 & 105.8 \\
\hline Non-mucous membranes & 1,702 & $767.6 \pm 749.3$ & 179.0 & 521.0 & $1,184.5$ & $1,005.5$ \\
\hline Head & 835 & $292.7 \pm 486.9$ & 29.5 & 111.5 & 375.3 & 345.8 \\
\hline Forehead & 80 & $26.4 \pm 74.8$ & 0.0 & 6.5 & 18.8 & 18.8 \\
\hline Chin & 326 & $195.6 \pm 323.4$ & 28.0 & 85.0 & 160.5 & 132.5 \\
\hline Cheek & 372 & $224.8 \pm 434.3$ & 17.3 & 51.5 & 119.8 & 102.5 \\
\hline Ear & 89 & $28.1 \pm 44.1$ & 1.5 & 8.0 & 45.0 & 43.5 \\
\hline \multicolumn{7}{|c|}{ Contact duration per contact (sec/contact) ${ }^{3}$} \\
\hline Mucous membranes & 1,305 & $5.0 \pm 3.0$ & 2.9 & 3.9 & 6.0 & 3.1 \\
\hline Eye & 180 & $4.7 \pm 3.3$ & 3.0 & 4.1 & 5.1 & 2.1 \\
\hline Nose & 541 & $4.4 \pm 3.7$ & 2.4 & 3.4 & 4.5 & 2.1 \\
\hline Mouth & 584 & $5.9 \pm 3.5$ & 2.4 & 4.5 & 6.5 & 4.0 \\
\hline Non-mucous membranes & 1,702 & $13.1 \pm 11.3$ & 6.3 & 8.2 & 17.7 & 11.4 \\
\hline Head & 835 & $9.3 \pm 10.1$ & 3.8 & 5.7 & 10.1 & 6.2 \\
\hline Forehead & 80 & $8.0 \pm 9.1$ & 2.7 & 3.5 & 9.3 & 6.6 \\
\hline Chin & 326 & $13.6 \pm 8.3$ & 8.5 & 13.2 & 17.7 & 9.2 \\
\hline Cheek & 372 & $17.3 \pm 43.1$ & 4.2 & 6.0 & 9.6 & 5.4 \\
\hline Ear & 89 & $11.0 \pm 19.1$ & 2.4 & 5.0 & 10.8 & 8.4 \\
\hline \multicolumn{7}{|c|}{ Contact exposure (frequency-duration/sec/person) ${ }^{4}$} \\
\hline Mucous membranes & 1,305 & $12,920.5 \pm 18,978.9$ & $4,087.0$ & $5,795.0$ & $12,553.5$ & $8,466.5$ \\
\hline Eye & 180 & $314.0 \pm 489.2$ & 14.0 & 57.5 & 444.5 & 430.5 \\
\hline Nose & 541 & $1,833.4 \pm 3,204.0$ & 312.8 & 600.0 & $2,361.8$ & $2,049.0$ \\
\hline Mouth & 584 & $5,309.6 \pm 14,691.7$ & 128.0 & $1,356.0$ & $3,360.5$ & $3,232.5$ \\
\hline Non-mucous membranes & 1,702 & $63,290.6 \pm 89,051.4$ & $5,860.8$ & $25,683.0$ & $79,055.3$ & $73,194.5$ \\
\hline Head & 835 & $17,433.8 \pm 45,438.6$ & 222.0 & $1,415.0$ & $14,162.3$ & $13,940.3$ \\
\hline Forehead & 80 & $231.1 \pm 897.9$ & 0.0 & 13.0 & 72.0 & 72.0 \\
\hline Chin & 326 & $5,824.4 \pm 17,158.5$ & 120.0 & 523.5 & $1,845.8$ & $1,725.8$ \\
\hline Cheek & 372 & $6,903.3 \pm 21,456.1$ & 40.5 & 331.0 & $1,978.5$ & $1,938.0$ \\
\hline Ear & 89 & $157.1 \pm 302.4$ & 1.5 & 18.0 & 134.3 & 132.8 \\
\hline
\end{tabular}

SD, standard deviation; $1 \mathrm{Q}, 25$ th percentile; $3 \mathrm{Q}$, 75th percentile; IQR, interquartile range.

'Contact frequency is the average number of hand-to-face contacts per person for 2-hours observation.

${ }^{2}$ Contact duration is the average second of hand-to-face contacts per person for 2 -hours observation.

${ }^{3}$ Contact duration per contact $=2 /$.

${ }^{4}$ Contact exposure $\left({ }^{1} x^{2}\right)$ was defined by multiplying the contact frequency by the contact duration. 

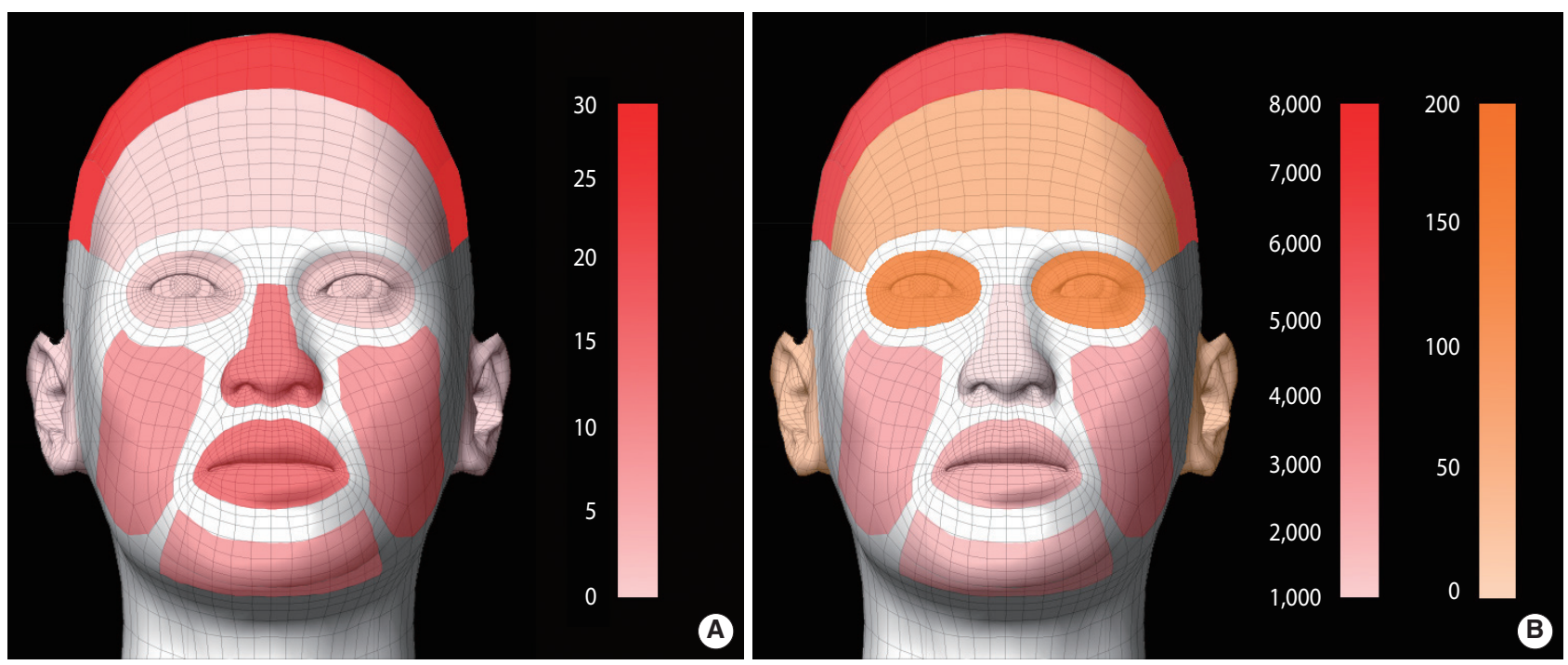

Figure 1. Visualization of the frequency and contact exposure of hand-to-face contact. (A) Visualization of the frequency of hand-to-face contact. The color bar expresses the contact frequency on a scale from 0 to 30; the darker the color, the more frequent the contact. (B) Visualization of hand contact exposure to the face. The more contact exposure (frequency-duration/sec/person), the darker the color, ranging from orange to red.

\section{Descriptive statistics of self-contact and hand-to-face contact}

The descriptive statistics of HFC are shown in Table 2. A total of 30 participants were observed, making 3,007 HFCs during 2 hours of observation. Of the HFCs, $43.4 \%$ (1,305 contacts) involved contact with mucous membranes such as the eyes, nose, and mouth.

The median frequency (n/person) of HFCs per person was highest for the mouth (16.5), followed by the nose (15.5) and head (13.0). The median CD (sec/person) of mucous membrane HFCs per person was longest for the head (111.5), followed in order by the chin (85.0), mouth (72.5), cheek (51.5), and nose (42.5). The median CD per contact (sec/contact) of mucous membrane HFCs was the longest for the mouth (4.5), followed by the eye (4.1) and nose (3.4). The median CD per contact (sec/ contact) of non-mucous membrane HFCs was the longest for the chin (13.2), followed by the cheek (6.0) and head (5.7). The mean values of $\mathrm{CF}, \mathrm{CD}$, and $\mathrm{CE}$ are also presented in Table 2, and the rankings of frequency according to mean values were consistent with those obtained using median values (Table 2).

The median CE (/sec/person) for contacts involving mucous membranes was the longest for the mouth $(1,356.0)$, followed by the nose (600.0) and eye (57.5). The median CE for non-mucous membrane contacts was the longest for the head $(1,415.0)$, followed by the chin (523.5) and cheek (331.0). Figure 1 visualizes the HFC frequency and exposure.

\section{Differences in contact indices of hand-to-face contact by the setting of indoor activities}

Table 3 shows that there was no significant difference in the median $\mathrm{CF}$ of the mucous membrane (eye, nose, and mouth) HFCs according to different indoor settings. The median CF of non-mucous membrane HFCs was different for head contact (higher in the classroom setting) $(\mathrm{p}<0.001)$. The median $\mathrm{CD}$ for mucous membrane contact with the mouth was significantly higher among the participants in classroom settings $(\mathrm{p}=0.026)$. The median $\mathrm{CE}$ was significantly higher for the mouth $(\mathrm{p}=0.037)$ and head $(\mathrm{p}<0.001)$ among the participants in the classroom setting.

\section{DISCUSSION}

This pilot study conducted to describe the frequency, duration, and intensity of HFC among Korean adults is the first study of HFC conducted in Korea. The results of this study improve our understanding of HFC patterns among Korean adults during indoor activities in daily life and provide evidence for estimating exposure to pathogens via HFC, thereby furnishing strong support for hand hygiene and the need to avoid HFC.

Participants' age varied widely because they were selected from adults $\geq 20$ years of age to include age groups broadly representative of the adult population.

In this study, almost half of the HFCs involved contact with mucous membranes (eye, nose, and mouth). This finding is consistent with a previous study by Kwok et al. [4] who reported a proportion of mucosal contact of $44.0 \%$ and non-mucosal contact of $56.0 \%$.

The average CF of HFC in this study was higher than those reported in previous studies: 23/person/hr [4], and 15.7/person/hr [5], 9.5/person/hr [9], and 2.6/person/hr [10]; however, our results are consistent with the finding of 27.7 (6-49)/45 min of another study [12]. The most frequently encountered non-mucous membrane and mucous membrane HFC areas in this study were 
Table 3. Differences in the contact indices of hand-to-face contact by the settings of indoor activities ( $n=30,2$-hour observation time)

\begin{tabular}{|c|c|c|c|c|}
\hline \multirow{2}{*}{ Variables } & \multicolumn{3}{|c|}{ Indoor activity settings } & \multirow{2}{*}{$\begin{array}{c}\text { p-value } \\
\text { (post-hoc) }{ }^{4}\end{array}$} \\
\hline & Chapel $(n=10)^{1}$ & Classroom $(n=10)^{2}$ & Office $(n=10)^{3}$ & \\
\hline \multicolumn{5}{|l|}{ Contact frequency (n/person) $)^{5}$} \\
\hline Mucous membranes & $39.5[33.0 ; 45.0]$ & $54.5[39.0 ; 79.0]$ & $35.5[22.0 ; 49.0]$ & 0.148 \\
\hline Eye & $3.0[2.0 ; 10.0]$ & $3.5[3.0 ; 6.0]$ & $5.0[2.0 ; 12.0]$ & 0.624 \\
\hline Nose & $10.5[8.0 ; 18.0]$ & $13.0[10.0 ; 18.0]$ & $16.0[15.0 ; 21.0]$ & 0.307 \\
\hline Mouth & $16.5[12.0 ; 27.0]$ & $20.5[14.0 ; 48.0]$ & $6.0[2.0 ; 18.0]$ & 0.054 \\
\hline Non-mucous membranes & $25.0[12.0 ; 32.0]$ & $73.0[63.0 ; 106.0]$ & $53.0[26.0 ; 103.0]$ & $0.001(2,3>1)$ \\
\hline Head & $5.5[4.0 ; 10.0]$ & $50.5[17.0 ; 62.0]$ & $11.5[8.0 ; 35.0]$ & $0.001(2,3>1)$ \\
\hline Forehead & $0.0[0.0 ; 2.0]$ & $2.0[0.0 ; 4.0]$ & $3.0[1.0 ; 8.0]$ & 0.146 \\
\hline Chin & $3.0[2.0 ; 6.0]$ & $7.0[5.0 ; 27.0]$ & $7.0[5.0 ; 17.0]$ & 0.067 \\
\hline Cheek & $6.5[3.0 ; 14.0]$ & $5.5[3.0 ; 10.0]$ & $13.0[4.0 ; 22.0]$ & 0.332 \\
\hline Ear & $1.5[0.0 ; 2.0]$ & $3.0[1.0 ; 8.0]$ & $2.5[1.0 ; 4.0]$ & 0.189 \\
\hline \multicolumn{5}{|l|}{ Contact duration (sec/person) ${ }^{6}$} \\
\hline Mucous membranes & $119.0[100.0 ; 188.0]$ & $267.5[184.0 ; 324.0]$ & $152.0[110.0 ; 237.0]$ & 0.076 \\
\hline Eye & $16.5[5.0 ; 74.0]$ & $18.0[12.0 ; 35.0]$ & $20.5[6.0 ; 41.0]$ & 0.961 \\
\hline Nose & $40.5[30.0 ; 66.0]$ & $36.5[28.0 ; 104.0]$ & $100.0[34.0 ; 147.0]$ & 0.239 \\
\hline Mouth & $48.5[18.0 ; 110.0]$ & $123.5[92.0 ; 224.0]$ & $23.5[7.0 ; 70.0]$ & $0.026(2>1,3)$ \\
\hline Non-mucous membranes & $175.0[92.0 ; 201.0]$ & $1,182.0[617.0 ; 2091.0]$ & $587.0[407.0 ; 1362.0]$ & $0.001(2,3>1)$ \\
\hline Head & $26.0[15.0 ; 66.0]$ & $424.0[210.0 ; 599.0]$ & $88.0[37.0 ; 381.0]$ & $<0.001(2>1,3)$ \\
\hline Forehead & $0.0[0.0 ; 7.0]$ & $9.0[0.0 ; 22.0]$ & $18.5[2.0 ; 70.0]$ & $0.034(3>1,2)$ \\
\hline Chin & $26.0[8.0 ; 111.0]$ & $114.5[59.0 ; 391.0]$ & $80.5[57.0 ; 226.0]$ & 0.153 \\
\hline Cheek & $44.5[17.0 ; 110.0]$ & $46.0[18.0 ; 59.0]$ & $94.0[9.0 ; 466.0]$ & 0.780 \\
\hline Ear & $3.0[0.0 ; 11.0]$ & $35.5[9.0 ; 74.0]$ & $6.0[1.0 ; 36.0]$ & 0.053 \\
\hline \multicolumn{5}{|c|}{ Contact duration per contact (sec/contact) ${ }^{7}$} \\
\hline Mucous membranes & $3.6[2.5 ; 4.7]$ & $4.8[3.8 ; 6.6]$ & $3.5[2.8 ; 7.7]$ & 0.188 \\
\hline Eye & $5.0[4.2 ; 5.7]$ & $4.7[3.6 ; 4.8]$ & $3.1[2.5 ; 4.0]$ & 0.096 \\
\hline Nose & $3.5[2.3 ; 4.6]$ & $3.4[2.6 ; 3.9]$ & $3.3[2.3 ; 9.2]$ & 0.830 \\
\hline $\begin{array}{l}\text { Mouth } \\
\text { continued }\end{array}$ & $2.9[2.1 ; 4.5]$ & $5.4[4.8 ; 9.0]$ & $4.4[2.3 ; 6.2]$ & 0.064 \\
\hline Non-mucous membranes & $6.2[5.5 ; 6.7]$ & $14.9[7.3 ; 25.4]$ & $11.4[7.2 ; 17.6]$ & $0.020(2,1>3)$ \\
\hline Head & $5.3[3.5 ; 5.7]$ & $9.6[5.7 ; 21.1]$ & $5.9[2.8 ; 12.3]$ & 0.054 \\
\hline Forehead & $2.7[1.7 ; 3.4]$ & $3.5[2.7 ; 7.7]$ & $6.2[3.7 ; 20.7]$ & 0.094 \\
\hline Chin & $8.7[4.0 ; 13.3]$ & $16.2[12.6 ; 21.0]$ & $13.2[11.2 ; 14.5]$ & 0.141 \\
\hline Cheek & $6.4[3.5 ; 8.5]$ & $6.0[5.0 ; 9.6]$ & $5.5[3.0 ; 19.3]$ & 0.627 \\
\hline Ear & $3.2[2.2 ; 3.7]$ & $9.2[7.2 ; 13.8]$ & $2.4[2.0 ; 5.6]$ & $0.025(2>1,3)$ \\
\hline \multicolumn{5}{|c|}{ Contact exposure (frequency-duration/sec/person) ${ }^{8}$} \\
\hline Mucous membranes & $5,191.5[4,000.0 ; 6,642.0]$ & $13,497.0[6,560.0 ; 29,160.0]$ & $5,153.0[3,960.0 ; 12,462.0]$ & 0.076 \\
\hline Eye & $49.5[10.0 ; 740.0]$ & $63.5[36.0 ; 252.0]$ & $107.0[12.0 ; 492.0]$ & 0.827 \\
\hline Nose & $427.5[270.0 ; 912.0]$ & $450.0[240.0 ; 1872.0]$ & $2,358.5[544.0 ; 2,820.0]$ & 0.113 \\
\hline Mouth & $756.0[252.0 ; 2,025.0]$ & $2,059.0[1,656.0 ; 9,216.0]$ & $132.5[8.0 ; 1,260.0]$ & $0.037(2>1,3)$ \\
\hline Non-mucous membranes & $5,169.5[1,104.0 ; 6,432.0]$ & $83,483.5[43,190.0 ; 115,346.0]$ & $39,077.0[12,168.0 ; 77,634.0]$ & $<0.001(2>3>1)$ \\
\hline Head & $172.0[55.0 ; 738.0]$ & $16,496.0[7,849.0 ; 26,950.0]$ & $855.5[318.0 ; 14,946.0]$ & $<0.001(2>1,3)$ \\
\hline Forehead & $0.0[0.0 ; 14.0]$ & $28.0[0.0 ; 60.0]$ & $53.0[2.0 ; 256.0]$ & 0.065 \\
\hline Chin & $72.0[12.0 ; 1020.0]$ & $766.5[236.0 ; 12,121.0]$ & $693.0[330.0 ; 4,294.0]$ & 0.148 \\
\hline Cheek & $331.0[34.0 ; 1,540.0]$ & $252.0[54.0 ; 605.0]$ & $1692.0[36.0 ; 8,388.0]$ & 0.506 \\
\hline Ear & $4.5[0.0 ; 33.0]$ & $103.5[9.0 ; 592.0]$ & $18.0[1.0 ; 96.0]$ & 0.084 \\
\hline
\end{tabular}

Values are presented as median [25th percentile; 75 th percentile].

'Indoor activities in chapel congregational worship.

${ }^{2}$ Indoor extracurricular activities in the classroom in a university.

${ }^{3}$ Indoor administrative computer work, lecture, discussion, and writing activities in the offices in a university.

${ }^{4}$ The Kruskal-Wallis test and post hoc test were pairwise comparisons using the Dunn test for multiple comparisons of independent samples using the Bonferroni adjustment method for $p$-values.

${ }^{5}$ Contact frequency is the average number of hand-to-face contacts per person for 2-hours observation.

${ }^{6}$ Contact duration is the average second of hand-to-face contacts per person for 2-hours observation.

${ }^{7}$ Contact duration per contact $=6 / 5$.

${ }^{8}$ Contact exposure $\left({ }^{5} x^{6}\right)$ was defined by multiplying the contact frequency by the contact duration. 
the head and the mouth, nose, and eyes, respectively. This is consistent with a previous study (head and mouth: $4 / \mathrm{hr}$, nose: $3 / \mathrm{hr}$, and eyes: $3 / \mathrm{hr}$ ) [4], the head was reported as the most frequent contact area [14], and mouth contacts occurred twice as often as nose or eye contacts [9]. However, this finding is inconsistent with results reporting the nose as the highest contact area (44.9\%) in biosafety level-2 workers [10]. The most frequent HFC area of the face has been reported to vary according to nationality, with British people frequently touching the chin and mouth, while Japanese people frequently touched the nose and eyes [15]. Since HFC studies have reported various results in different study populations, further research will be needed to develop effective preventive measures regarding HFC for Koreans.

The average CD of HFCs (sec/contact) was highest for the head, followed in order by the mouth, nose, and eyes. This order corresponds to that of a previous report, but with longer durations [4]. Among mucous membrane contacts, the mouth was confirmed to have the highest CE index, followed by the nose.

In the comparative analysis of the 3 group settings, the indices of CF, CD, and CE were higher for the mouth and head in the college student group in classroom settings than other two groups in other settings, which is consistent with the characteristics of HFC observed in young adults $[4,16]$. Further research will be needed to identify differences in HFC according to age groups and different settings of indoor activities.

In this study, HFC was more frequent and longer than observed in previous studies $[4,9,10]$. The mouth showed the highest CF, CD, and CE of HFC, followed by the nose. The rate of HFC in this study is sufficient to predict respiratory disease acquisition according to Nicas \& Best [5]. These results suggest that the mouth and nose may be major exposure routes of self-inoculation in Korean adults. In addition to the mouth $[17,18]$, particular attention should be paid to the nasal mucosa as a source of respiratory infections [3], especially given its large number of resident microbes, and it is associated with a high risk of spreading infections to other parts of the nasal passages via the hands [19]. Therefore, this study's results will be useful for reducing the risk of respiratory tract infections by informing efforts to reduce HFC to the nose $[3,20]$. Though not as risky as HFC to mucous membranes, non-mucous membrane HFC of the head and cheek should also be avoided because the face can become contaminated if touched with unwashed hands, and this may subsequently enable the spread of pathogens to mucous membranes via HFC [21].

The CF or CD indices were useful for comparing the findings of the present study with those of previous studies reporting CF or $\mathrm{CD}[4,5,9,10,14,15]$. However, these indices alone have limitations in terms of estimating the total exposures via HFC. The CE index was more useful for differentiating and clearly distinguishing between mouth and nose exposures via HFC than the simpler indices of $\mathrm{CF}$ or $\mathrm{CD}$ of the mouth and nose alone, and therefore more useful for estimating the exposure risk via HFC.

In addition, the results of this study could be used as strong evi- dence for the importance of following good respiratory hygiene, which involves regularly and thoroughly cleaning one's hands, avoiding touching one's eyes, nose, and mouth with one's hands, and covering one's mouth and nose with a bent elbow or tissue when coughing or sneezing [22].

Education is needed to raise awareness of the risks of HFC, and strategies need to be developed to reduce habitual HFC behaviors $[20,21,23]$. The risk of infectious disease transmission through HFC can be prevented through the modification of HFC behaviors [23]. Furthermore, during a pandemic (such as the coronavirus disease 2019 [COVID-19] pandemic), the use of masks can be a part of a comprehensive package for prevention and control measures in different settings [24]. These precautions can protect the mouth and nose, which were identified as possible routes for infectious microbe inoculations via hand contact.

This study had some limitations. It measured HFCs during indoor activities within a limited time period with a limited number of participants, and was the first such study in Korea. Furthermore, it used convenience sampling. Therefore, it was not possible to analyze all HFCs over a 24-hour period or capture every situation, and therefore the generalizability of the findings to the entire Korean population is limited. Further studies will be required for higher generalizability. Nevertheless, this study described in detail the characteristics of HFC for the first time, and the findings can be used as effective evidence of self-inoculation via HFC and provide evidence for good hand hygiene and avoiding HFC. Furthermore, the results of this study will be useful for developing exposure models and control strategies to prevent infectious disease transmission via HFC, especially during pandemics such as COVID-19.

In conclusion, this study showed that the CF and CD of HFC were more frequent and longer than reported in previous studies. The most frequent HFC exposure area was the mouth, followed by the nose. Therefore, the mouth and nose may be the most frequent exposure routes for infectious pathogens. The CE index may be more useful than other indices for measuring total exposure via HFC. Avoiding habitual HFC to the mouth and nose, building awareness of self-inoculation via HFC, and following good respiratory hygiene should be promoted to prevent exposure to pathogens. Furthermore, studies on HFC in various situations, specific age groups, and larger populations will be needed to generalize the findings to the Korean population.

\section{CONFLICT OF INTEREST}

The authors have no conflicts of interest to declare for this study.

\section{FUNDING}

This research was supported by research funding from the Korea Disease Control and Prevention Agency (grant No. 2017P140300). 


\section{ACKNOWLEDGEMENTS}

The authors wish to thank the study participants for their contribution to the research. The authors would also like to thank YS Kim and MJ Shin for their assistance with data collection.

\section{AUTHOR CONTRIBUTIONS}

Conceptualization: HSO. Data curation: HSO, YY. Formal analysis: MR. Funding acquisition: HSO. Methodology: HSO. Project administration: HSO. Visualization: HSO. Writing - original draft: HSO, MR, YY. Writing - review \& editing: HSO, MR, YY.

\section{ORCID}

Hyang Soon Oh: https://orcid.org/0000-0002-5709-2861; Mikyung Ryu: https://orcid.org/0000-0002-9915-3175; Youngran Yang: https://orcid.org/0000-0001-5610-9310

\section{REFERENCES}

1. Boyce JM, Pittet D; Healthcare Infection Control Practices Advisory Committee; HICPAC/SHEA/APIC/IDSA Hand Hygiene Task Force. Guideline for Hand Hygiene in Health-Care Settings. Recommendations of the Healthcare Infection Control Practices Advisory Committee and the HIPAC/SHEA/APIC/IDSA Hand Hygiene Task Force. Am J Infect Control 2002;30:S1-S46.

2. Pittet D, Allegranzi B, Boyce J; World Health Organization World Alliance for Patient Safety First Global Patient Safety Challenge Core Group of Experts. The World Health Organization Guidelines on Hand Hygiene in Health Care and their consensus recommendations. Infect Control Hosp Epidemiol 2009;30:611-622.

3. Fukunaka Y, Sawada SS, Nishiura C, Noguchi J, Tsukamoto K, Otsuji Y, et al. Susceptibility to upper respiratory tract infection and touching of the eyes or nose: a cross-sectional study of Japanese workers. J Occup Health 2013;55:66-73.

4. Kwok YL, Gralton J, McLaws ML. Face touching: a frequent habit that has implications for hand hygiene. Am J Infect Control 2015; 43:112-114.

5. Nicas M, Best D. A study quantifying the hand-to-face contact rate and its potential application to predicting respiratory tract infection. J Occup Environ Hyg 2008;5:347-352.

6. Macias AE, de la Torre A, Moreno-Espinosa S, Leal PE, Bourlon MT, Ruiz-Palacios GM. Controlling the novel A (H1N1) influenza virus: don’t touch your face! J Hosp Infect 2009;73:280-281.

7. Gwaltney JM Jr, Moskalski PB, Hendley JO. Hand-to-hand transmission of rhinovirus colds. Ann Intern Med 1978;88:463-467.

8. Kuramoto-Chikamatsu A, Honda T, Matsumoto T, Shiohara M, Kawakami Y, Yamauchi K, et al. Transmission via the face is one route of methicillin-resistant Staphylococcus aureus cross-infection within a hospital. Am J Infect Control 2007;35:126-130.

9. Elder NC, Sawyer W, Pallerla H, Khaja S, Blacker M. Hand hygiene and face touching in family medicine offices: a Cincinnati Area Research and Improvement Group (CARInG) network study. J Am Board Fam Med 2014;27:339-346.

10. Johnston JD, Eggett D, Johnson MJ, Reading JC. The influence of risk perception on biosafety level-2 laboratory workers' hand-toface contact behaviors. J Occup Environ Hyg 2014;11:625-632.

11. Hendley JO, Wenzel RP, Gwaltney JM Jr. Transmission of rhinovirus colds by self-inoculation. N Engl J Med 1973;288:1361-1364.

12. Lewis RC, Rauschenberger R, Kalmes R. Hand-to-mouth and other hand-to-face touching behavior in a quasi-naturalistic study under controlled conditions. J Toxicol Environ Health A 2021;84: 49-55.

13. Centers for Disease Control and Prevention. How to protect yourself \& others; 2020 [cited 2020 Jul 1]. Available from: https://www. cdc.gov/coronavirus/2019-ncov/prevent-getting-sick/prevention. html.

14. Wilson AM, Verhougstraete MP, Beamer PI, King MF, Reynolds KA, Gerba CP. Frequency of hand-to-head, -mouth, -eyes, and -nose contacts for adults and children during eating and non-eating macro-activities. J Expo Sci Environ Epidemiol 2021;31:34-44.

15. Hatta T, Dimond SJ. Differences in face touching by Japanese and British people. Neuropsychologia 1984;22:531-534.

16. Zhang N, Jia W, Wang P, King MF, Chan PT, Li Y. Most self-touches are with the nondominant hand. Sci Rep 2020;10:10457.

17. Gorman Ng M, Davis A, van Tongeren M, Cowie H, Semple S. Inadvertent ingestion exposure: hand- and object-to-mouth behavior among workers. J Expo Sci Environ Epidemiol 2016;26: 9-16.

18. Sahmel J, Hsu EI, Avens HJ, Beckett EM, Devlin KD. Estimation of hand-to-mouth transfer efficiency of lead. Ann Occup Hyg 2015;59:210-220.

19. Whitby M, McLaws ML, Ross MW. Why healthcare workers don't wash their hands: a behavioral explanation. Infect Control Hosp Epidemiol 2006;27:484-492.

20. Maeda A. Can not touching the nose or eyes help cold prevention? Possibility of application using a smartwatch and self-checking. Annu Int Conf IEEE Eng Med Biol Soc 2020;2020:5722-5728.

21. Heinicke MR, Stiede JT, Miltenberger RG, Woods DW. Reducing risky behavior with habit reversal: a review of behavioral strategies to reduce habitual hand-to-head behavior. J Appl Behav Anal 2020;53:1225-1236.

22. World Health Organization. Coronavirus disease (COVID-19) advice for the public; 2021 [cited 2021 Mar 22]. Available from: https://www.who.int/emergencies/diseases/novel-coronavirus2019/advice-for-public.

23. Mueller SM, Martin S, Grunwald M. Self-touch: contact durations and point of touch of spontaneous facial self-touches differ depending on cognitive and emotional load. PLoS One 2019;14: e0213677.

24. World Health Organization. Guidance on mask use in the context of COVID-19 [cited 2021 Mar 21]. Available from: https:// openwho.org/courses/COVID-19-mask-use. 